\title{
Complete countrywide mortality in COVID patients receiving ECMO in Germany throughout the first three waves of the pandemic
}

\author{
Christian Karagiannidis ${ }^{1 *} \mathbb{D}$, Arthur S. Slutsky ${ }^{2}$, Thomas Bein ${ }^{3}$, Wolfram Windisch', Steffen Weber-Carstens ${ }^{4}$ and \\ Daniel Brodie ${ }^{5}$
}

\section{Research Letter}

Extracorporeal membrane-oxygenation (ECMO) has been widely used for COVID-19-related acute respiratory distress syndrome (ARDS), with a mortality rate of $37.1 \%$ based on the largest published series [1]. This rate is comparable to ECMO-supported patients with nonCOVID-19-related ARDS [1]. However, some reports suggest that the real-life mortality is higher than that reported above [2], including a cohort of 768 COVID-19 patients in Germany with an in-hospital mortality rate of $73 \%$ [3], and a surprising finding is given that health care resources in Germany were not notably under strain during the pandemic. To better characterize this discrepancy, we evaluated in-hospital mortality for all COVID19 patients in Germany supported with venovenous ECMO (VV-ECMO).

We report unbiased and unselected follow-up data at hospital discharge from the federal German hospital payment institute (InEK) of all 3.397 COVID-19 patients supported with VV-ECMO in Germany from March $1^{\text {st }}$, 2020, through May $31^{\text {st }}, 2021$. ECMO in Germany was

*Correspondence: Christian.Karagiannidis@uni-wh.de: karagiannidisc@kliniken-koeln.de

${ }^{1}$ Department of Pneumology and Critical Care Medicine, ARDS and ECMO Centre, Cologne-Merheim Hospital, Kliniken Der Stadt Köln gGmbH, Witten/ Herdecke University Hospital, Ostmerheimer Strasse 200, 51109 Cologne, Germany

Full list of author information is available at the end of the article provided in 213 intensive care units (ICUs) - out of a total of 1.684 intensive care units across 1.288 hospitals (www.intensivregister.de). The study was approved by the Ethics Committee of the Witten/Herdecke University.

The mean age of all ECMO patients remained stable over the study period ( $57 \pm 11$ years). As expected, survivors were younger than the non-survivors (53 years (range $47-63$ y) vs. 59y (Range: 54-63y)), independent of timing during the pandemic. The mean duration of ECMO support was not different between survivors and non-survivors, averaging 17 days. Overall, in-hospital mortality was $68 \%$. Of note, the mortality for any given week (Fig. 1) shows some degree of variation, as survivors spend more time in hospital than non-survivors.

In the largest unbiased and unselected real-life cohort reported to date of VV-ECMO-supported patients with COVID-19-related respiratory failure, we found that inhospital mortality for patients in Germany was $68 \%$. This is markedly higher than that reported from other countries or from international registries [2-5], confirming prior results [3] of a higher mortality rate in Germany than reported elsewhere.

Mortality in ECMO-supported COVID-19 patients may be increasing over the course of the pandemic, as reported in the most recent Extracorporeal Life Support Organization (ELSO) cohort with a mortality of $51.9 \%$ in patients initiated on ECMO after May 1, 2020 [5]. However, that is still lower than the present cohort from Germany. One explanation is the older mean age of 


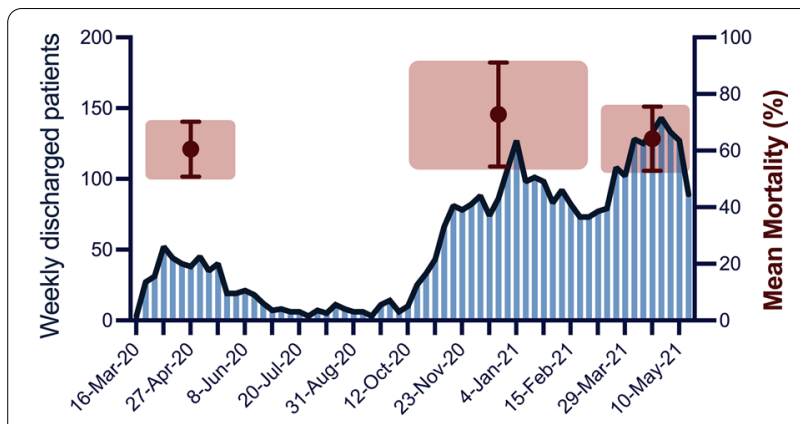

Fig. 1 Weekly patient number of patients being treated with W-ECMO in Germany for COVID-19-related acute respiratory failure between March 2020 and May 2021. Mean mortality is given for all three waves with mean and standard deviation

$57 \pm 11$ years in our report compared to the ELSO report (median 51 years). Another explanation is that the use of ECMO in Germany is not centrally regulated and clinicians could elect to initiate ECMO without constraints from regulatory bodies, potentially extending criteria beyond those patients who would be likely to benefit. Furthermore, in Germany, there is an incentive system for the control of health care which is characterized by proportional reimbursement: "as more procedures are done, as more will be paid by insurances," probably leading to a mixture of 'true' indications and reimbursement temptations [6]. A comprehensive central case registry would be particularly important during a pandemic to frequently analyze data and draw updated conclusions. Finally, the ELSO data represent dedicated ECMO centers, whereas the current data are unselected from all German hospitals.

A major strength of the study is inclusion of every COVID-19 patient treated with VV-ECMO in Germany (population $\sim 83,000,000$ ) over the course of the study. One limitation is the lack of granularity of the data, including comorbidities, impairing our ability to better understand the reasons for the high mortality.

Ultimately, the present data should serve as a warning to clinicians. Even in a country with adequate resources, mortality in ECMO-supported patients with COVID19-related respiratory failure may be high if its use is not restricted to patients deemed most likely to benefit.

\section{Acknowledgements}

We are very grateful to the federal German hospital payment institute (InEK, Institut für das Entgeltsystem im Krankenhaus) for their outstanding work.

\section{Authors' contributions}

CK is responsible for the data integrity and analyzed the data together with all authors. CK and DB wrote the main draft of the manuscript. All authors had full access to the data. All authors contributed to interpretation and writing of the manuscript and approved the final manuscript.
Availability of data materials

Not applicable.

\section{Declarations}

\section{Ethics approval and consent to participate}

The study was approved by the Ethics Committee of the Witten/Herdecke University.

\section{Consent for publication}

All authors approved the final draft of the manuscript and gave their consent for publication.

\section{Competing interests}

CK reports personal fees from Maquet, personal fees from Xenios, personal fees from Bayer, non-financial support from Speaker of the German register of ICUs, grants from the German Ministry of Research and Education, during the conduct of the study. TB, WW and SWC have nothing to disclose. AS reports personal fees from Baxter and Xenios in relation to consulting with respect to ECMO. DB receives research support from ALung Technologies. He has been on the medical advisory boards for Baxter, Abiomed, Xenios and Cellenkos.

\section{Author details}

${ }^{1}$ Department of Pneumology and Critical Care Medicine, ARDS and ECMO Centre, Cologne-Merheim Hospital, Kliniken Der Stadt Köln gGmbH, Witten/ Herdecke University Hospital, Ostmerheimer Strasse 200, 51109 Cologne, Germany. ${ }^{2}$ Keenan Research Centre for Biomedical Science, Li Ka Shing Knowledge Institute, St Michael's Hospital; University of Toronto, Toronto, Canada. ${ }^{3}$ Faculty of Medicine, University of Regensburg, Regensburg, Germany. ${ }^{4}$ Department of Anesthesiology and Operative Intensive Care Medicine (CCM, CVK), Charité - Universitätsmedizin Berlin, Berlin, Germany. ${ }^{5}$ Department of Medicine, Columbia University College of Physicians and Surgeons, and the Center for Acute Respiratory Failure, New York-Presbyterian Hospital, New York, USA.

Received: 29 October 2021 Accepted: 17 November 2021

Published online: 29 November 2021

\section{References}

1. Ramanathan K, Shekar K, Ling RR, et al. Extracorporeal membrane oxygenation for COVID-19: a systematic review and meta-analysis. Crit Care. 2021;25(1):211.

2. Lebreton G, Schmidt M, Ponnaiah M, et al. Extracorporeal membrane oxygenation network organisation and clinical outcomes during the COVID-19 pandemic in Greater Paris, France: a multicentre cohort study. Lancet Respir Med 2021.

3. Karagiannidis C, Strassmann S, Merten M, et al. High in-hospital mortality in COVID patients receiving ECMO in Germany - A critical analysis. Am J Respir Crit Care Med 2021.

4. Broman LM, Eksborg S, Coco VL, et al. Extracorporeal membrane oxygenation for COVID-19 during first and second waves. Lancet Respir Med 2021.

5. Barbaro RP MG, Boonstra PS, Combes A, Agerstrand CL, Annich G, Diaz R, Fan E, Hryniewicz K, Lorusso R, Paden ML, Stead CM, Swol J, Iwashyna TJ, Slutsky AS, Brodie D; for the Extracorporeal Life Support Organization. Extracorporeal membrane oxygenation for COVID-19: Evolving outcomes from the international Extracorporeal Life Support Organization Registry. Lancet 2021; (in press August 19).

6. Friedrichson B, Mutlak H, Zacharowski K, Piekarski F. Insight into ECMO, mortality and ARDS: a nationwide analysis of 45,647 ECMO runs. Crit Care. 2021;25(1):38.

\section{Publisher's Note}

Springer Nature remains neutral with regard to jurisdictional claims in published maps and institutional affiliations. 